\title{
Clinical Evidence of Vestibular Dysregulation in Colicky Babies Before and After Chiropractic Treatment Versus Non-Colicky Babies.
}

Jan Hoeve ( $\sim$ janhoeve@chiropractiestaphorst.nl )

Wethouder Bronstraat

Research article

Keywords: Infantile colic, vestibular, dysregulation, clinical index, neuromodulation

Posted Date: December 4th, 2020

DOl: https://doi.org/10.21203/rs.3.rs-117516/v1

License: (c) (i) This work is licensed under a Creative Commons Attribution 4.0 International License.

Read Full License 


\section{Abstract}

\section{Background}

After 65 years of research that has been primarily directed at differentiating between normal and colicky crying, the cause of infantile colic remains elusive and no definitive cure has been found. Given the general absence of pathology, colicky crying is widely considered the extreme end of a spectrum of normal crying behavior. In the literature scattered evidence can be found suggesting that infantile colic may be the behavioral expression of physiological brainstem dysregulation, particularly of the vestibular and autonomic nuclei. The purpose of this study is to present a five-point clinical index of vestibular (hyper)activity and its application to investigate vestibular dysregulation in colicky and non-colicky babies.

Methods

120 consecutive colicky babies were evaluated using this index, before and after a very gentle vibratory treatment, and compared to 117 non-colicky babies.

Results

Before treatment, of 120 colicky babies only $2(1.7 \%)$ scored 0 , whereas $118(98.3 \%)$ scored scored 1-5. Of 117 non-colicky babies $89(76,1 \%)$ scored 0 and 28 (23.9\%) scored 1-3, none scored 4-5. The odds ratio is OR ( $\mathrm{Cl}$ 95\%) 187.54 (43.52-808.09). After treatment 111 (92.5\%) scored 0 and 9 (7.5\%) scored 1-3, none scored 4-5. A McNemar test showed the difference before and after to be significant $\left(\chi^{2}=109.00, p\right.$ $<.001$ ). For colicky babies the mean vestibular score is 2.88 (SD 1.22), compared to 0.37 (SD 0.73) for non-colicky babies, a difference of $87.2 \%$. After treatment the score decreased from 2.88 (SD 1.12) to 0.10 (SD 0.40), or $96.5 \%$.

\section{Conclusion}

Colicky babies are not just infants who cry a lot. They also show clinical evidence of vestibular dysregulation. Gentle treatment aimed at relaxing tight sub-occipital musculature by means of a vibrational technique may be effective in decreasing vestibular hyperactivity, signifying an improvement in brainstem regulation. The vestibular index opens the prospect for development into a tool towards an objective and practical clinical diagnosis of infantile colic.

\section{Background}

Infantile colic is defined as excessive and inconsolable crying and fussing in an otherwise healthy and well-fed infant who is less than 5 months old. It is considered a benign behavioral disorder with a prevalence of $15-25 \%$ that spontaneously resolves after 4-5 months [1]. The diagnosis is commonly based on the (modified) Wessel criteria of crying for at least 3 hours a day during at least 3 days in the preceding week [1][2]. To date, after 65 years and a great deal of research the cause of colic remains 
elusive and no distinct cures have emerged. The one thing that has become clear, however, is that usually there is no pathology [3]. This has led some authors to suggest that colicky crying may just be the extreme end of normal crying behavior. Yet, the possibility of some underlying physiological dysregulation has remained largely unexplored.

Babies function at a basic level of visceral brainstem reflexes, because higher inhibitory modulating structures are not sufficiently developed yet [4]. Although little research has been done in this area, symptoms commonly associated with colic can be found scattered throughout the literature. These include: (1) asymmetric posture (C-curve) and head preference [5] even while asleep, which may lead to developmental plagio/brachiocephaly; (2) extensor hypertonicity and pseudo-opisthotonic posture [5][6]; (3) upper-cervical movement/joint dysfunction, muscular tightness and occipital tenderness [7]; (4) high levels of stress and stress arousal [6]; (5) breastfeeding difficulties [7][8][9][10]; (6) gastro-intestinal disorders such as regurgitation / Ger(d) and intestinal cramps with or without obstipation or dyschesia[11]. Together the associated symptoms point to dysregulation of the vestibular and autonomic systems, suggesting that infantile colic may be the behavioral expression of dysregulation at the brainstem level.

This present study deals with a clinical assessment of the vestibular system. A five-point clinical index of vestibular (hyper)activity is presented and applied as a tool to evaluate brainstem dysregulation in colicky babies before and after chiropractic treatment compared to non-colicky babies.

\section{Clinical index of vestibular (hyper)activity}

The index was developed in our chiropractic clinic where it has been utilized for over three years. Mild rhythmic vestibular stimulation is known to have a relaxing, soothing effect [12], whereas vestibular overstimulation tends to be uncomfortable and may lead to dizziness, nausea or even vomitting [13]. Therefore, if a baby does not react well to mild vestibular stimulation this could be taken as a possible sign of vestibular hyperactivity.

Our clinical index is comprised of five items, each consisting of a statement that can be answered by a simple agree/disagree. Each "agree" earns one point and each "disagree" earns zero points. So, the babies may have a score between $0-5$. The statements are based on literature reports, personal observations and reported parental experiences:

(A). Your baby does not calm down or fall asleep during a car ride. It is a common practice all over the world that babies are rocked in a cradle or in a carrying sling to help them calm down and fall asleep. Riding in a car may have a similar soothing effect as non-colicky babies generally fall asleep during a car trip. Some desperate parents take their colicky baby on evening car rides in the hope that they may stop screaming. In many cases this seems to work, but not always as available research is inconclusive. Some parents report that the baby relaxes only while the car is moving, but that with every stop at a traffic light the screaming starts again. This suggests that motion and not just car vibration or white noise is 
important to induce a calming effect. If the baby does not calm down or fall asleep during a car ride this is taken as a possible sign of vestibular hyperactivity.

(B). Your baby does not calm down or fall asleep when held against your chest or cradled in the crook of your arm, while you are walking around at a brisk pace. When a mouse or a cat mother wants to move her young she takes them in her mouth and carries them to the new spot. The little pups react with a mammalian calming reflex that is characterized by going limp, by a decrease in ultrasonic vocalizations (in mice) and a decrease in heart rate. These changes are mediated by the parasympathetic system and the cerebellum and in mice are dependent on tactile and proprioceptive stimuli [14]. Human babies show a similar calming response to carrying i.e. a decrease in voluntary movement, heart rate and crying [14]. However, the observation that the response is much stronger when the mother is walking at a brisk pace than when she is just sitting and holding the baby suggest that vestibular stimulation is at least equally important. When asked, many mothers of colicky babies deny that their infant cries excessively (for nobody wants a colicky baby or a cry baby). Upon further questioning they may tell that they have to carry their baby and walk around almost all day and that, if they do not, the baby will cry and be restless. This agrees with Esposito's [14] observation that the calming response stops the moment the carrying ends. If the baby does not calm down when held against the mother's chest while she is walking at a brisk pace this is taken as another possible sign of vestibular hyperactivity.

(C). When your baby has fallen asleep against your chest you cannot lay the baby supine in the crib without the baby waking up and crying. This item also relates to item (E). A common observation by parents is that colicky babies are not comfortable lying on their back. When held upright against the parent's chest, particulary if the parent is leaning backwards, the baby may be relatively calm or even fall asleep, but as soon as the baby is even gently laid down the screaming starts within a few minutes (without showing the characteristics of the Moro reflex). Some parents resort to spending the night sitting partially upright in bed with the baby sleeping against their chest. If the sleeping baby cannot be laid down supine in a crib without waking up this is taken as a sign of possible vestibular hyperactivity, because the only difference between the two positions is the orientation with respect to gravity.

(D). When sleeping the baby may wake up with a scream, showing the symptoms of the Moro reflex. Many parents report that once their colicky baby is asleep the infant may suddenly wake up with a scream, showing the characteristics of the Moro reflex. Since this behavior is not induced by an external stimulus such as a loud noise and because the Moro reflex, in contrast to the Startle reflex, is essentially a vestibular reflex [15], this behavior is taken as a possible sign of vestibular hyperactivity. Some parents report that when carrying the baby on their arm while walking down the stairs the baby will scream with every descending step. Other mothers descibe that the baby will scream the moment they sit down on a chair in preparation for breastfeeding. Such observations suggest that in these babies the vestibular threshold to the Moro reflex may be set low. It is surmised that while asleep the baby may have a sudden falling sensation as if the floor is dropping away from underneath them. 
(E). The baby is much more comfortable lying inclined in a car seat than supine in a crib. This item also relates to item (C). Many parents report that the baby is much more relaxed and sleeps much better when lying inclined in a car seat (Maxi-Cosi) than supine in a crib or bed. This observation may also be a sign of vestibular hyperactivity, because when inclined at an angle of some 30 degrees the position of the lateral semicircular canal is approximately vertical and presumably less receptive to stimuli [30]. The other relatively stable position is at a forward tilt of some 30 degrees against the parents chest when the lateral canal is approximately horizontal.

\section{Methods}

Included in this study were 120 consecutive, unhappy babies (mean age 6.4(SD 4.64) weeks; range 1-35 weeks; male $68.3 \%$; female $31.7 \%$ ), who presented to our chiropractic clinic for infantile colic and were treated with a very gentle method aimed at relaxing tight occipital/upper-cervical musculature. Excluded were 5 babies who withdrew early from treatment for financial reason because they were not covered by health insurance. All fulfilled the modified Wessel criteria [1] of crying 3 hours a day for three days during the preceding week. The actual treatment involves the application of slight, pulsed vibrations at selected points in the occipital/upper-cervical region by means of a spring-loaded reflex device (J-Tech device, zero setting, tangentially applied; no impact, just slight vibrations), aimed at relaxing tight sub-occipital musculature. This device and similar ones are widely used in the chiropractic treatment of musculoskeletal conditions, albeit at a higher instrument setting. The use of a somewhat different vibrotactile device was reported in a medical study investigating the therapeutic effects of parasympathetic neuromodulation in rheumatoid arthritis patients by means of cutaneous stimulation applied to the cymba of the external ear [16]. In the present study two treatments per week were given for an average of two weeks i.e. an average of four treatments in all (Table 1). The treatment was terminated when (1) the parents reported satisfactory improvement in general well-being, the infant having changed from a highly stressed, screaming baby into a relaxed, happy child; and (2) the treating chiropractor was satisfied that the occipital/upper-cervical musculature had relaxed. As controls 117 non-colicky, happy babies (mean age 7.5(SD 3.72) weeks; range 3-22 weeks; male 47\%; female 53\%) were included, who presented to our clinic as part of an ongoing program offering free check-ups. All colicky babies were categorized in four age groups: 1-4 weeks, 5-8 weeks, 9-12 weeks, greater than 13 weeks (Table 1).

Colicky babies, before and after treatment, and non-colicky babies were evaluated for vestibular dysregulation by means of the above clinical index of vestibular (hyper)activity (Table 1; Figures 1 and 2).

\section{Statistical analysis}

The data were analyzed by means of binomial logistic regression and Chi-square tests. The results are given as $\mathrm{n}(\%)$, as odds ratio (OR) with $95 \% \mathrm{Cl}$ and as Means with SD. Statistical computations were made using SPSS.

\section{Results}


Before treatment of the 120 colicky babies only $2(1.7 \%)$ scored 0 , whereas $118(98.3 \%)$ had a score 1-5. In the control group of 117 non-colicky babies 89 (76,1\%) scored 0, none scored 4-5 and $28(23.9 \%)$ scored 1-3. The odds ratio is OR (Cl 95\%) 187.54 (43.52-808.09). After treatment 111 (92.5\%) scored 0 , none scored 4-5 and 9 (7.5\%) scored 1-3 (Table 1; Figures 1 and 2). A McNemar test showed the difference before and after to be significant $\left(X^{2}=109.00, p<.001\right)$. Colicky babies before treatment had a mean vestibular score of 2.88 (SD 1.12), compared to 0.37 (SD 0.73) for non-colicky babies, a difference of $87.2 \%$. After treatment the score had decreased from 2.88 (SD 1.22) to 0.10 (SD 0.40 ), a drop of $96.5 \%$ (Table 1; Figure 1 and 2). The number of treatments given in each of the four age categories were closely similar at 4.40(SD 0.987), 4.59(SD 0.91); 4.53(SD 0.624) and 4.09(SD 0.701), respectively (Table 1) and, therefore, independent of age. For each age category the vestibular scores before and after treatment were: $1-4$ weeks 2.70 (SD 1.085) and 0.60(SD 0.233), respectively, or $88 \%$ improvement; 5-8 weeks 3.10 (SD 1.095) and 0.10(SD 0.384) or 97\% improvement; 9-12 weeks 2.76(SD 1.147) and 0.52(SD 0.850) or $83 \%$ improvement; greater than 13 weeks 3.00 (SD1.265) and 0.18 (SD 0.405 ) or $94 \%$ improvement. This means that the decrease in vestibular score is also independent of age.

\section{Discussion}

Much research in infantile colic has focussed on the excessive crying per se, as this is, at least for the parents, the most distressing aspect. A recent review established that normal crying in babies less than 3 months old averages 2 hours per day during the first two weeks, increases slightly to 2 hours 15 minutes at week 6 and then decreases to 1 hour 10 minutes by week 12 [1]. Nonetheless, there is a wide variation in crying time, some babies cry as little as half an hour a day, whereas others cry for many, many hours.

Because in infantile colic there is no recognizable pathology some authors have suggested that there is nothing wrong with these babies and that colic is just the extreme end of the spectrum of normal crying behavior. However, this is unlikely. When babies cry they convey a message to the parents that they are not comfortable and that their needs are to be met, be these physiological or emotional [17]. Since a baby's crying has a high emotional impact the parents find it difficult to ignore [18][19]. Normally this is fine because it compels the parents to tend to the baby's needs, and once these have been met, the crying and fussing stops. From an evolutionary perspective it is unlikely that the baby would engage in nonfunctional crying, because in order for the system to work the baby should "never cry wolf". From an energy perspective it is also unlikely because the excessive crying costs up to 20 times more energy compared to the quiet sleeping state [20]. That energy would be better spent on growing, rather than wasted on crying for no reason. Other authors have pointed to the dynamics of family relationships and sub-optimal infant-parent interaction as a possible cause of colicky behavior [22]. This too is unlikely because it cannot account for the common observation that one member of a pair of twins may be colicky whereas the other is not (personal observations in our clinic). It seems then that instead of accepting the premise that all babies cry, we should consider that babies do not cry unless they have a good reason to do so i.e. signaling discomfort or need and calling upon the parents to look after them. This puts infantile colic in a different perspective. If crying is a graded non-specific signal of distress [21] 
the question becomes one of why is it that such a high percentage of babies are so thoroughly uncomfortable and in so much distress (15-25\%).

The present study reveals that colicky babies are not just infants who cry a lot. They also show clinical evidence of underlying vestibular hyperactivity. The reason for this hyperactivity may be inferred from the realization that the vestibular system is modulated by upper-cervical proprioception and by the visual system [23][24]. In young infants, however, the visual system cannot play a significant role yet as visual gaze and and the vestibulo-ocular reflex are still immature [25][26][27]. For this reason vestibular modulation in small babies may primarily depend on upper-cervical proprioception. Afferent proprioceptive input from upper-cervical segments (C1-3) and from axial structures project to the vestibular nuclei and indirectly to the Purkinje cells of the medial cerebellar cortex. These Purkinje cells are inhibitory to the fastigial output nucleus, allowing the medial cerebellum to exert an inhibitory modulating influence on the vestibular system [28][29][30]. A state of vestibular hyperactivity may be facilitated by even slight, subclinical, diminished inhibition by the cerebellum [13]. Consequently, aberrant proprioceptive traffic into the vestibular system and the medial cerebellum arising from tight sub-occipital musculature, possibly aquired at birth [5], could conceivably provide a mechanism for dysregulation and hyperactivity of the vestibular nuclei. Conversely, treatment aimed at relaxation of the tight musculature may be expected to restore regular proprioceptive flow and facilitate normalization of the inhibitory modulation of the vestibular nuclei by the medial cerebellum.

In the present study gentle relaxation of upper-cervical tight musculature by means of a vibratory technique was associated with $96.5 \%$ decrease in the vestibular score, indicating a decrease in vestibular hyperactivity and an improvement of vestibular regulation. In the absence of a control group of untreated colicky babies this decrease cannot be conclusively attributed to the treatment that was administered. The observed decrease, however, is unlikely to reflect the natural course of colic as a self-limiting disorder, because the number of treatments required as well as the decrease of the vestibular score for all four age categories turned out to be closely similar and not dependent on age (Table 1). Moreover, after two weeks of treatment the babies in category 1-4 weeks were 3-6 weeks old. Considering that this is the peak age of infantile colic [1], it is clear that the decrease cannot reflect the natural course of the disorder. Therefore, it seems likely that the documented decrease in vestibular score may derive from the treatment that was applied. If so, this would suggest that central neuromodulation may be accomplished not only by cutaneous vibratory stimulation to cymba of the external ear [16], but also by similar stimulation of suboccipital musculature, at least in babies.

The vestibular system arises early in the embryological development. In concordance with their central role in many regulatory processes the vestibular nuclei have extensive projections to other brainstem nuclei [30]. This means that any vestibular dysregulation present may well be propagated to those nuclei, particulary of the autonomic system [13], leading to a spectrum of associated symptoms such as listed in the introduction. 
Strengths of the present study are that the five-point vestibular index is easily applied in a clinical setting and that a significant $87.2 \%$ difference between colicky and non-colicky babies could be documented. In current practice the diagnosis of infantile colic is generally one of exclusion. The vestibular index presented here, if confirmed and validated, could be developed into a tool that allows a clinical diagnosis to be made on the basis of distinct and objective criteria.

A weakness is the absence of a proper control group of untreated colicky babies. However, in a clinical setting where parents present their babies with the intention that they are treated, selection of a control group would be unethical and also impossible to achieve. With regards to future research it is suggested that the direction should be broadened from being almost exclusively focussed on the excessive crying per se, to also include the sympoms of possible dysregulation at the brainstem level.

\section{Conclusions}

Colicky babies are not just infants who cry a lot. They also show clinical evidence of underlying vestibular dysregulation i.e. dysregulation at a brainstem level. Gentle treatment aimed at correcting upper-cervical muscular tightness by means of a vibratory technique may be effective in decreasing vestibular hyperactivity, signifying an improvement of brainstem regulation. The clinical vestibular index presented here offers the potential for development into a tool towards an objective and practical diagnosis of infantile colic.

\section{Abbreviations}

$\mathrm{Cl}$ is confidence interval; SD is standard deviation

Gerd is Gastro-esophagial reflux disorder.

\section{Declarations}

\section{Ethics approval and consent to participate}

Data were collected as part of regular treatments and check-ups. The data were extracted from patient files. Under Dutch law no approval of an ethics committee is required.

All individuals who present at our clinic for treatment or check-up are invited to give their written informed consent (a) for treatment, and/or (b) that a report may be sent to other health professional, (c) that their data may be used anonymously for research purposes and (d) incorporated in scientific publications. For all babies included in the present study parents or guardians gave their written consent.

\section{Consent for publication}

All parents or guardians of participating babies gave their written informed consent that their data may anonymously be used in this study and incorporated in a scientific publication. 
Availability of data and materials

The datasets used and/or analyzed during the current study are available from the corresponding author on reasonable request.

\section{Competing interest}

The author declares that he has no competing interests.

\section{Authors' contribution}

The author is solely responsible for all aspects of this publicaion.

\section{Funding}

There was no external funding.

\section{Acknowledgement}

I am grateful to my colleaques Fleur de Haan, Kathrine Sund and Igor Dijkers for stimulating discussions and critical revieuws of early drafts of the manuscript. Statistical computations were provided by M. van Leerdam of Barestatistics. Logistic support was provided by G. de Weert.

\section{References}

1. Wolke, D., A. Bilgin, and M. Samara, Systematic Review and Meta-Analysis: Fussing and Crying Durations and Prevalence of Colic in Infants. J Pediatr. 2017. 185: p. 55-61 e4.

2. Wessel, M.A., et al., Paroxysmal fussing in infancy, sometimes called colic. Pediatrics. 1954. 14(5): p. 421-35..

3. Freedman, S.B., N. Al-Harthy, and J. Thull-Freedman, The crying infant: diagnostic testing and frequency of serious underlying disease. Pediatrics. 2009. 123(3): p. 841-8.

4. Jouen, F., Head position and posture in newborn infants. In: Head-neck sensory motor system, Berhoz, A., Graf W. and Vidal P. (Eds); Oxford University Press. 1992

5. Biedermann, H., Manual therapy in children: proposals for an etiologic model. J Manipulative Physiol Ther. 2005; 28(3): p. e1-15.

6. Levine, M.I., Emotional factors in the etiology of colic in infants. Pediatrics. 1956. 18(5): p. 836-8

7. Miller-Loncar, C., et al., Infant colic and feeding difficulties. Arch Dis Child. 2004. 89(10): p. 908-12.

8. Zwart, P., M.G. Vellema-Goud, and P.L. Brand, Characteristics of infants admitted to hospital for persistent colic, and comparison with healthy infants. Acta Paediatr. 2007. 96(3): p. 401-5.

9. Douglas, P.S. and P.S. Hill, A neurobiological model for cry-fuss problems in the first three to four months of life. Med Hypotheses. 2013. 81(5): p. 816-22. 
10. Degenaar, H. and A. Kritzinger, Suck, swallow and breathing coordination in infants with infantile colic. S Afr J Commun Disord. 2015. 62(1): p. e1-e10.

11. Benninga, M.A., et al., Childhood Functional Gastrointestinal Disorders: Neonate/Toddler. Gastroenterology. 2016.

12. Vrugt, D.T. and D.R. Pederson, The effects of vertical rocking frequencies on the arousal level in twomonth-old infants. Child Dev. 1973. 44(1): p. 205-9.

13. Bernetti, L., et al., Subclinical vestibular dysfunction in migraineurs without vertigo: A Clinical study. Acta Neurol Scand. 2018. 138(4): p. 270-277.

14. Esposito, G., et al., Infant calming responses during maternal carrying in humans and mice. Curr Biol. 2013. 23(9): p. 739-45.

15. Futagi, Y., Y. Toribe, and Y. Suzuki, The grasp reflex and moro reflex in infants: hierarchy of primitive reflex responses. Int J Pediatr. 2012. 2012: p. 191562.

16. Addorisio, M.E., et al., Investigational treatment of rheumatoid arthritis with a vibrotactile device applied to the external ear. Bioelectron Med. 2019. 5: p. 4.

17. Zeifman, D.M., An ethological analysis of human infant crying: answering Tinbergen's four questions. Dev Psychobiol. 2001. 39(4): p. 265-85.

18. Lorberbaum, J.P., et al., Feasibility of using fMRI to study mothers responding to infant cries. Depress Anxiety. 1999. 10(3): p. 99-104.

19. Riem, M.M.E., et al., As tears go by: Baby tears trigger more brain activity than adult tears in nulliparous women. Soc Neurosci. 2017. 12(6): p. 633-636.

20. Wells, J.C., Parent-offspring conflict theory, signaling of need, and weight gain in early life. Q Rev Biol. 2003. 78(2): p. 169-202.

21. Gustafson, G., R. Wood and J. Green, Can we hear the causes of infants' crying,in: R.G. Barr, B. Hopkins, J.A. Green (Eds) Crying as a sign, a symptom. A signal. MacKeith Press/Cambridge University Press, London. 2000:8-22

22. Raiha, H., et al., Excessively crying infant in the family: mother-infant, father-infant and mother-father interaction. Child Care Health Dev. 2002. 28(5): p. 419-29.

23. Gdowski, G.T. and R.A. McCrea, Neck proprioceptive inputs to primate vestibular nucleus neurons. Exp Brain Res. 2000. 135(4): p. 511-26.

24. Sprenger, A., et al., Postural Control in Bilateral Vestibular Failure: Its Relation to Visual, Proprioceptive, Vestibular, and Cognitive Input. Front Neurol. 2017. 8: p. 444.

25. Sarnat, H.B., Functions of the corticospinal and corticobulbar tracts in the human newborn , Journal of pediatric neurology. 1(1):3-8, 2003.

26. Weissman, B.M., A.O. DiScenna, and R.J. Leigh, Maturation of the vestibulo-ocular reflex in normal infants during the first 2 months of life. Neurology. 1989. 39(4): p. 534-8. 
27. Finocchio, D.V., K.L. Preston, and A.F. Fuchs, Infant eye movements: quantification of the vestibuloocular reflex and visual-vestibular interactions. Vision Res. 1991. 31(10): p. 1717-30.

28. Pompeiano, O., Vestibulospinal relations: vestibular influences on gamma motoneurons and primary afferents. Prog Brain Res. 1972. 37: p. 197-232.

29. De Zeeuw, C.I. and A.S. Berrebi, Postsynaptic targets of Purkinje cell terminals in the cerebellar and vestibular nuclei of the rat. Eur J Neurosci. 1995. 7(11): p. 2322-33.

30. Beck, R., Functional neurology for practitioners of Manual Therapy: Churchill Livingstone. ISBN-13: 978-043-10220-2, 2008.

\section{Tables}


Table 1: Characteristics colicky versus non-colicky babies

Colic $n=120$

Non-colic $n=117$

\begin{tabular}{|lll|}
\hline Age & $6.37(4.84)$ & $7.45(3.72)$ \\
\hline Male & $68.3 \%$ & $47.0 \%$ \\
\hline Female & $31.7 \%$ & $53.0 \%$ \\
\hline
\end{tabular}

Total vestibular score

Colic Before Colic After Non-colic

\begin{tabular}{|c|c|c|c|c|c|}
\hline Score $=0, n(\%)$ & 02 & $(01.67)$ & \multicolumn{2}{|c|}{ 112(93.33) } & 89 (76.07) \\
\hline Score $=1, \mathrm{n}(\%)$ & 10 & $(08.33)$ & 6 & $(5.00)$ & $15(12.82)$ \\
\hline Score $=2, n(\%)$ & 31 & $(25.83)$ & 1 & $(0.83)$ & $11(9.40)$ \\
\hline Score $=3, \mathrm{n}(\%)$ & 47 & (39.17) & 1 & $(0.83)$ & $2(1.71)$ \\
\hline Score $=4, \mathrm{n}(\%)$ & 19 & (15.83) & 0 & (0) & $0 \quad(0)$ \\
\hline score $=5, \mathrm{n}(\%)$ & 11 & (09.17) & 0 & (0) & $0(0)$ \\
\hline Mean (SD) & 2.8 & $3(01.12)$ & & $(0.40)$ & $0.37(0.73)$ \\
\hline
\end{tabular}

Vestibular score by individual characteristics

\begin{tabular}{llll} 
& Colic Before & Colic After & Non-colic \\
\hline A - Car ride, n (\%) & $34(28.33)$ & $0(0)$ & $7(0.06)$ \\
\hline B - Carrying, n (\%) & $48(40.00)$ & $0(0)$ & $1(0.85)$ \\
\hline C - Laying down, n (\%) & $97(80.83)$ & $2(1.66)$ & $14(11.97)$ \\
\hline D - Moro, n (\%) & $96(80.00)$ & $4(3.33)$ & $12(10.26)$ \\
\hline E - Car seat, n (\%) & $71(58.33)$ & $1(0.83)$ & $9(7.69)$
\end{tabular}

\section{Total Vestibular score by age group}

\begin{tabular}{|llll|} 
& Colic before & Colic after & $\%$ decrease \\
\hline 1-4 weeks, Mean (SD) & $2.70(1.09)$ & $0.06(0.23)$ & 97.8 \\
\hline 5-8 weeks, Mean (SD) & $3.10(1.10)$ & $0.10(0.38)$ & 96.8 \\
\hline 9-12 weeks, Mean (SD) & $2.76(1.15)$ & $0.18(0.72)$ & 93.5 \\
\hline
\end{tabular}




\begin{tabular}{|lll|}
\hline$>13$ weeks, Mean (SD) & $3.00(1.27)$ & $0.18(0.40)$ \\
\hline 1-4 weeks, Mean (SD) & Treatments & 94.0 \\
\hline $5-8$ weeks, Mean (SD) & $4.59(0.98)$ \\
\hline 9-12 weeks, Mean (SD) & $4.53(0.62)$ \\
$>13$ weeks, Mean (SD) & $4.09(0.70)$ \\
\hline Number of treatments & $4.45(0.89)$ \\
\hline Odds Ratio colic/non-colic & $187.54(43.52-808.09)$ \\
\hline$\chi^{2}$ before /after & $109.00 \quad \mathrm{p}<.001$ \\
\hline
\end{tabular}

\section{Figures}

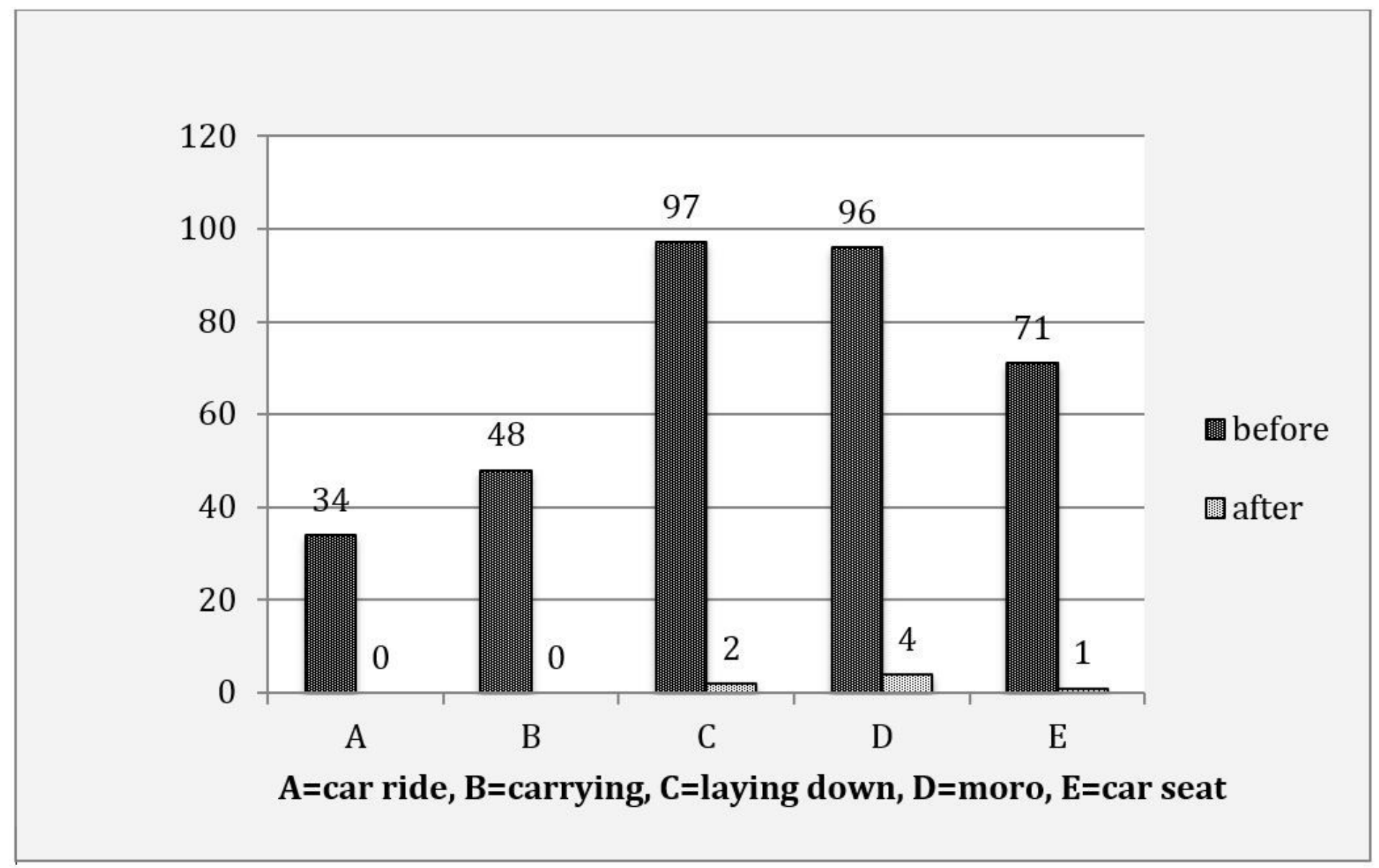

Figure 1

Colicky babies: Vestibular score by induvidual characteristics before and after treatment 


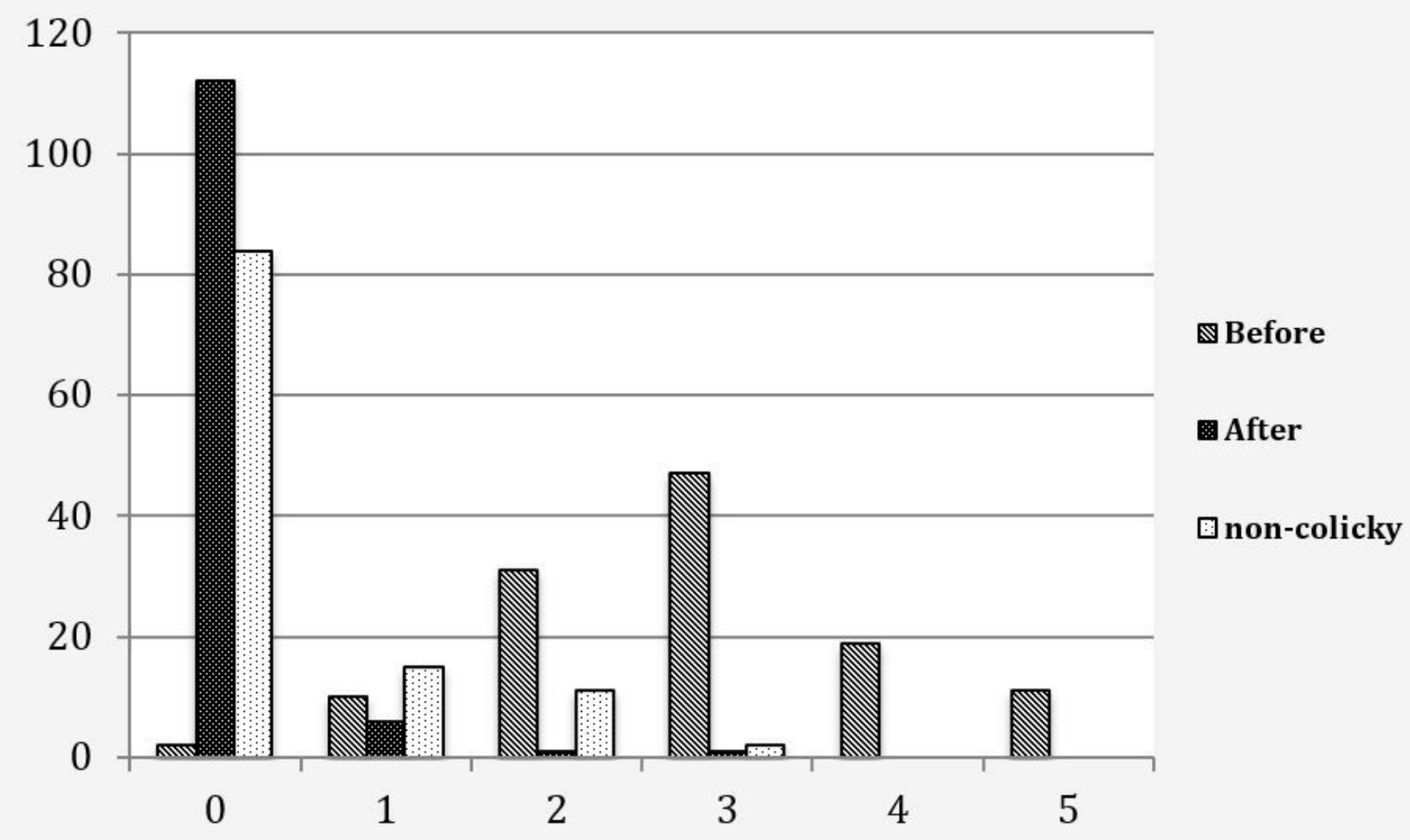

Figure 2

Colicky babies before and after treatment compared to non-colicky babies: total vestibular score 